\title{
DETERMINAÇÃO TEÓRICA DAS CONFORMAÇÕES MAIS ESTÁVEIS DA TREALOSE EM SOLUÇÃO AQUOSA
}

\author{
Cinthia S. Soares e Clarissa O. da Silva* \\ Departamento de Química, Universidade Federal Rural do Rio de Janeiro, Rodovia BR 465, km 47, 23890-000 Seropédica - \\ RJ, Brasil
}

Recebido em 29/1/07; aceito em 27/7/07; publicado na web em 19/12/07

\begin{abstract}
THEORETICAL DETERMINATION OF THE MOST STABLE TREHALOSE CONFORMATIONS IN AQUEOUS SOLUTION. In this work the most abundant trehalose conformers for the isolated molecule as well as for the water solvated system are selected. The theoretical tecniques employed are $a b$ initio calculations in the gas phase and in aqueous solution using the PCM model. A conformational map is built for the glycosidic angles $(\Phi$ and $\Psi)$ and the search for the most abundant structures is explained. The final structures are validated by the agreement found between experimental and theoretical values for ${ }^{3} \mathrm{~J}_{\mathrm{H}, \mathrm{C}}$ along the glycosidic linkage.
\end{abstract}

Keywords: trehalose conformation; disaccharide conformation; conformational map.

\section{INTRODUÇÃO}

Os carboidratos compõem a maior das quatro classes de biomoléculas, juntamente com proteínas, ácidos nucléicos e lipídeos. Esses compostos desempenham importantes funções biológicas, sendo os metabólitos principais em processos de reserva e produção de energia ${ }^{1}$.

Dentre os carboidratos do tipo dissacarídeo, que são formados por duas unidades monossacarídicas unidas através de uma ligação glicosídica, a trealose - um açúcar não-redutor, que consiste de duas moléculas de $D$-glicose unidas por uma ligação $\alpha-1,1$ - se destaca por uma função particular (Figura 1).

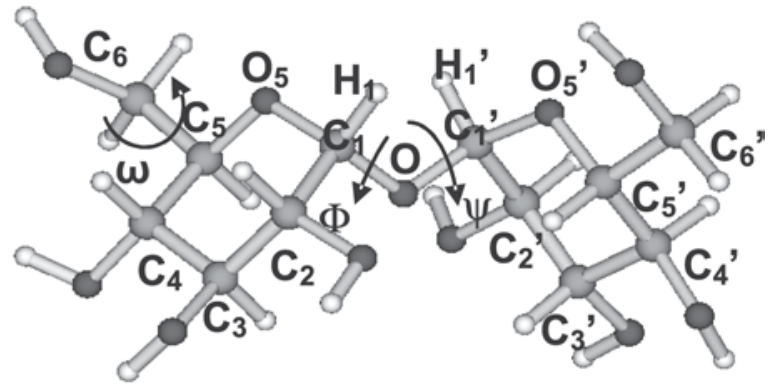

Figura 1. Trealose: $\alpha$-D-glicopiranosil- $(1 \rightarrow 1)$ - $\alpha$-D-glicopiranose, ângulos diedros $\Phi\left(O_{5}-C_{1}-O-C_{1}{ }^{\prime}\right)$ e $\Psi\left(C_{1}-O-C_{1}{ }^{\prime}-O_{5}{ }^{\prime}\right)$ da ligação glicosídica e $\omega$, que define a orientação do grupo hidroximetilênico

Este dissacarídeo é encontrado na natureza em microorganismos, plantas e invertebrados ${ }^{2}$. É bem conhecida a sua ação protetora em diversos tipos de estresses ambientais impostos a estes organismos que levam à desidratação ${ }^{3}$, e há estudos recentes sobre sua ação também como antioxidante ${ }^{4}$. A trealose é extensivamente utilizada nas áreas alimentícia, de cosméticos e na indústria médica em geral; mas é na tecnologia de conservação de drogas e biomateriais que se concentram os grandes interesses atuais. No entanto, a maior dificuldade no aprimoramento desta tecnologia deve-se ao desconhecimento do mecanismo molecular através do qual a trealose interage e estabiliza membranas e proteínas. Exis-

*e-mail: clarissa-dq@ufrrj.br tem algumas propostas para este mecanismo: Cerrutti et al. ${ }^{5}$ propuseram que o mecanismo de proteção tanto de membranas celulares como de proteínas acontecesse através de vitrificação. Mais recentemente, Pereira et al. ${ }^{6}$ e Sum et al. ${ }^{7}$, através de estudos de dinâmica molecular, parecem propor que a interação entre a trealose e a membrana fosfolipídica aconteça preferencialmente através de interações tipo ligação hidrogênio, que se tornam mais intensas à medida que o sistema perde água. Entretanto, não se pode precisar ainda ao certo se a trealose substitui a água normalmente presente no organismo em seu estado não desidratado, ou aprisiona esta, impedindo que seja eliminada para o meio durante a desidratação. Nem mesmo um estudo quantitativo da energia de estabilização decorrente da interação trealose-membrana, quando comparada àquela estabelecida entre a membrana e outros dissacarídeos, foi ainda realizado ${ }^{8-10}$.

Assim sendo, pretendeu-se investigar, num primeiro momento, as conformações mais abundantes da trealose em solução aquosa para, a partir destas, e de um protótipo de membrana também em desenvolvimento em nosso grupo, propor um possível mecanismo de interação para o sistema trealose-membrana fosfolipídica, explorando a relação estrutura-atividade e quantificando a energia de estabilização desta interação.

\section{PARTE EXPERIMENTAL}

Neste trabalho foi utilizada metodologia ab initio em nível Hartree-Fock (HF) com um conjunto de funções de base do tipo 6$31 \mathrm{G}(\mathrm{d}, \mathrm{p})$, que se mostrou adequado ao estudo conformacional de monossacarídeos em estudos anteriores ${ }^{11}$, dedicados exclusivamente à seleção de métodos convenientes para amostragens conformacionais em superfícies de energia potencial para carboidratos.

A utilização de metodologia $a b$ initio para sistemas desta dimensão, e com tantas possibilidades conformacionais, poderia a princípio ser desencorajada ${ }^{12}$ não fosse a necessidade da correta descrição das interações que determinam tais conformações. Estas interações são basicamente de três tipos: ligação hidrogênio, efeito anomérico/exo-anomérico e interações não ligadas ${ }^{13}$, sendo, dificilmente, quantitativamente bem descritas por outras técnicas, como mecânica molecular ${ }^{14,15}$.

Como mencionado anteriormente, carboidratos são moléculas 
que se apresentam à temperatura ambiente numa grande variedade de conformações. No estudo conformacional de dissacarídeos, a primeira informação relevante refere-se à orientação relativa das unidades monossacarídicas, que vem estabelecida pelo valor dos ângulos diedros $\Phi$ e $\Psi$ que definem a ligação glicosídica (Figura 1). Estes são os parâmetros geométricos determinantes para a energia de uma dada conformação ${ }^{12,13}$.

Neste trabalho, partiu-se de uma estrutura inicial construída para este sistema e otimizada através de mecânica molecular com campo de força Dreiding $\mathrm{II}^{16}$. Tal estrutura foi posteriormente otimizada em nível HF/6-31G(d,p). A geometria obtida foi utilizada na construção do mapa conformacional para a molécula isolada, através da varredura dos ângulos diedros glicosídicos, com 12 passos de $30^{\circ} \mathrm{em}$ cada ângulo. Cada estrutura obtida por rotação de cada um destes parâmetros foi completamente otimizada e, assim, após 144 otimizações de geometria foi construído um mapa conformacional relaxado completo em nível HF/6-31G(d,p) para a trealose a partir da interpolação destes dados. Nesta etapa do trabalho utilizou-se o programa Jaguar v6.0 $0^{17}$.

A segunda etapa do trabalho constituiu-se da otimização de todos os parâmetros geométricos, salvo $\Phi$ e $\Psi$ que permaneceram fixados nos valores obtidos para as regiões de maior estabilidade no mapa conformacional. Todos os cálculos descritos a partir desta etapa foram executados no programa Gaussian 2003, em sua versão B.04 ${ }^{18}$.

Numa terceira etapa, partindo-se das geometrias da etapa precedente, otimizaram-se todos os parâmetros geométricos, relaxando assim por completo a geometria de cada confôrmero. Estas estruturas foram solvatadas e novamente otimizadas, conforme descrito a seguir. Ao final desta etapa, passou-se então à investigação da orientação dos grupos hidroximetilênicos para os confôrmeros mais estáveis obtidos na etapa anterior. Todas as 9 possibilidades (GG, GT e TG) foram consideradas (três para cada unidade monossacarídica), para cada par de ângulos glicosídicos. Ao final desta etapa, foi calculada a população de Boltzmann de cada confôrmero em solução aquosa.

As informações a respeito da geometria destas estruturas em solução aquosa foram obtidas com o modelo de solvatação Polarizable Continuum Model - $\mathrm{PCM}^{19}$. Tal modelo propõe tratar quanto-mecanicamente o soluto, enquanto o solvente é descrito como um contínuo de propriedades particulares. Nesta formulação, o soluto vem imerso neste contínuo através de uma cavidade nele aberta (Figura 2), construída pela interligação de esferas centradas em átomos ou grupos de átomos selecionados do soluto. Os raios destas esferas utilizados neste trabalho foram $2,28 \AA$ para os grupos $\mathrm{CH}$ ou $\mathrm{CH}_{2}, 1,80 \AA$ para os átomos de $\mathrm{O}$ e 1,44 A para os átomos de $\mathrm{H}$ hidrolizáveis.

Neste modelo a energia do sistema, auto-valor do operador hamiltoniano que contém o potencial de interação soluto-solvente ${ }^{20}$, tem status de energia livre, e se denomina energia livre em solução $\left(\mathrm{G}_{\mathrm{sol}}\right)$. Esta grandeza se decompõe em várias componentes, como
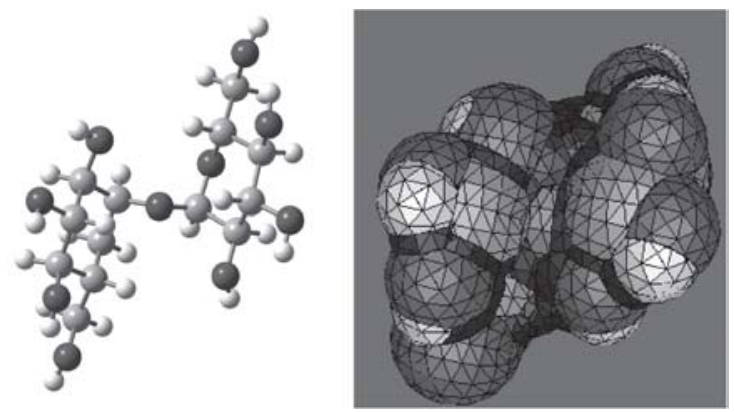

Figura 2. Cavidade para a trealose segundo o modelo de solvatação PCM indicado na Equação 1.

$\mathrm{G}_{\text {sol }}=\mathrm{G}_{\text {eletr }}+\mathrm{G}_{\text {cav }}+\mathrm{G}_{\text {rep-disp }}+\mathrm{G}_{\mathrm{MM}}$

onde $G_{\text {eletr }}, G_{\text {cav }}, G_{\text {rep-disp }}$ e $G_{\text {MM }}$ são, respectivamente, as componentes eletrostática, de cavitação, de repulsão-dispersão e de movimento molecular da energia livre em solução ${ }^{20}$, sendo a última componente $\left(\mathrm{G}_{\mathrm{MM}}\right)$ ignorada na versão do modelo PCM utilizada neste trabalho.

Para a validação das estruturas obtidas foram calculadas as constantes de acoplamento de spin heteronuclear $\left({ }^{3} \mathrm{~J}_{\mathrm{HC}}\right)$ para o segmento H-C-O-C da ligação glicosídica dos confôrmeros mais abundantes em solução aquosa, a partir da Equação de $\operatorname{Karplus}^{21}$ (Equação 2), onde $\Phi_{\mathrm{H} 1}$ é o valor do ângulo diedro em graus para o segmento H1C1-O-C1', e $\Phi_{\mathrm{H} 1^{\prime}}$ para o segmento C1-O-C1'-H1'(Figura 1).

${ }^{3} \mathbf{J}_{\mathrm{H}, \mathrm{C}}=5,7 \cos ^{2}\left(\Phi_{\mathrm{H}}\right)-0,6 \cos \left(\Phi_{\mathrm{H}}\right)+0,5$

Esta propriedade foi escolhida pelo fato de ser muito sensível a pequenas variações dos ângulos diedros e, adicionalmente, por depender exatamente dos ângulos glicosídicos $\Phi$ e $\Psi$, que constituem os parâmetros geométricos mais importantes para a energia de uma dada conformação.

\section{RESULTADOS E DISCUSSÃO}

A geometria de partida obtida para a construção do mapa conformacional abaixo apresentado possui como valores de ângulo diedro $\Phi=80^{\circ}$ e $\Psi=77^{\circ}$ (Figura 1).

No mapa conformacional da Figura 3, foram identificadas cinco regiões de energia mínima, denominadas A, B, C, D e E.

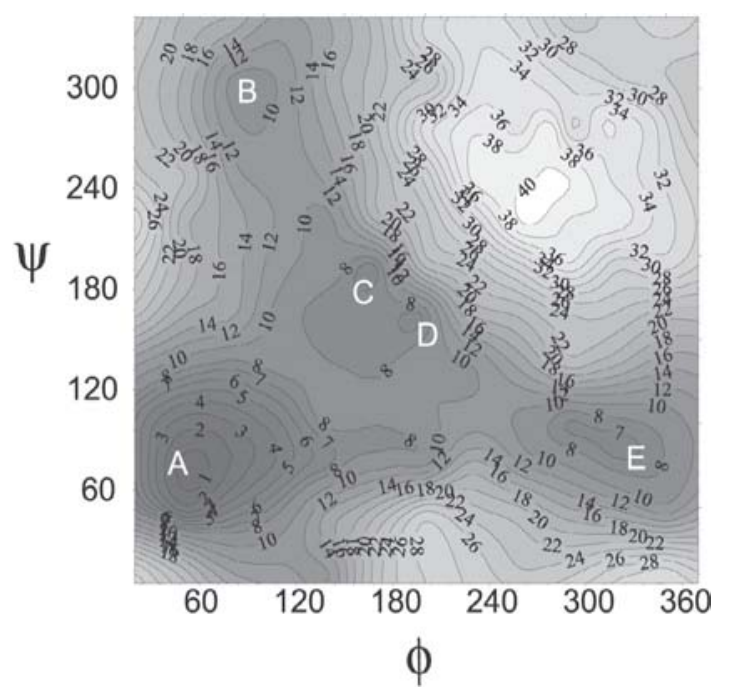

Figura 3. Mapa conformacional relaxado em nível HF/6-31G(d,p) em fase gasosa para a trealose - 144 pontos. Valores de energia em $\mathrm{kcal} / \mathrm{mol}$

A primeira etapa do trabalho constitui-se da otimização de todos os parâmetros geométricos, salvo $\Phi$ e $\Psi$ que permaneceram fixados nos valores obtidos a partir do mapa conformacional como anteriormente descrito. A Tabela 1 apresenta os resultados para estas otimizações.

Numa etapa posterior uma nova otimização dos parâmetros geométricos foi realizada - agora com todos os parâmetros geométricos livres. Cálculos de freqüência para estas estruturas foram realizados e as correções térmicas e entrópicas para os cinco confôrmeros foram introduzidas. Durante esta etapa, 3 confôrmeros convergiram 
Tabela 1. Confôrmeros com parâmetros geométricos otimizados (menos $\Phi$ e $\Psi$ ) e respectivas energias eletrônicas relativas (em $\mathrm{kcal} / \mathrm{mol})$

\begin{tabular}{lccc}
\hline Confôrmero & $\Phi$ & $\Psi$ & $\Delta \mathrm{U}_{\mathrm{T}=0 \mathrm{~K}}$ \\
\hline A & 71 & 55 & $+0,6$ \\
B & 65 & 92 & 0 \\
C & 171 & 162 & $+4,2$ \\
D & 161 & 170 & $+4,2$ \\
E & 89 & 42 & $+2,1$
\end{tabular}

Para o confôrmero B, $\mathrm{U}_{\mathrm{T}=0 \mathrm{~K}}=-1290,714082$ u.a., sendo $\Delta \mathrm{U}_{\mathrm{T}=0 \mathrm{~K}}=$ $\mathrm{U}_{\mathrm{T}=0 \mathrm{~K}}(\mathrm{X})-\mathrm{U}_{\mathrm{T}=0 \mathrm{~K}}(\mathrm{~B})$.

para as outras 2 estruturas e, assim, apenas 2 estruturas foram obtidas ao final deste processo: os conformeros A, B e E convergiram para uma única estrutura - denominada estrutura $\mathrm{A}$ e os confôrmeros $\mathrm{C}$ e D convergiram para uma outra estrutura - denominada estrutura C. Os resultados obtidos são mostrados na Tabela 2.

Os valores de população de Boltzmann $\left(\mathrm{p}_{\mathrm{i}}\right)$ destes confôrmeros em fase gasosa são exibidos na Tabela 2. É importante ressaltar que estes valores consideram somente as diferenças de energia para as posições relativas dos anéis monossacarídicos. Para este grau de liberdade, parece haver para o sistema isolado somente uma orientação estável.

Com o objetivo de determinar as geometrias adotadas pela trealose em solução aquosa, os cinco confôrmeros otimizados em fase gasosa foram solvatados segundo o modelo de contínuo polarizável - PCM e suas geometrias reotimizadas. A Tabela 2 também traz os dados desta etapa do trabalho. Como no caso anterior os confôrmeros A, B e E convergiram para uma única estrutura - denominada estrutura $\mathrm{A}$ e os confôrmeros C e D convergiram para uma outra estrutura - denominada estrutura C. Os valores de população de Boltzmann $\left(\mathrm{p}_{\mathrm{i}}\right)$ encontrados para os confôrmeros da trealose em solução aquosa estão indicados na última coluna da Tabela 2. Novamente, como em fase gasosa, parece haver somente uma orientação estável para os anéis monossacarídicos em solução aquosa que, aliás, pouco difere daquela obtida em fase gasosa. A partir destes resultados, pode-se propor que a conformação relativa dos anéis monossacarídicos para a trealose seja determinada por efeitos intramoleculares.

Em função destes resultados, seria importante neste ponto ter-se uma noção dos efeitos do solvente sobre a energia do sistema e sobre sua geometria (que claramente também afeta sua energia) de modo mais independente. Por esta razão, ainda na Tabela 2, apresentam-se os cálculos tipo "single point" realizados com o PCM, para a geometria dos confôrmeros A e C obtida para o sistema isolado (fase gasosa). Estes cálculos quantificam o efeito do solvente somente sobre a energia do sistema, sem considerar os efeitos geométricos.

Como podemos perceber pelos resultados obtidos, os efeitos do solvente sobre a geometria do sistema podem ser negligenciados, sem perda de informação relevante.

Esta constatação, a princípio, poderia surpreender, pois imagina-se que compostos polifuncionais como os carboidratos, com grupos que tendem a interagir fortemente com um solvente polar e prótico, como a água, poderiam se apresentar quando solvatados em conformações distintas daquelas dos sistemas isolados. Porém, mesmo para a glicose, se observa que a orientação das hidroxilas (cujas rotações têm barreiras com alturas muito inferiores àquela da ligação glicosídica) é invariante com o meio ${ }^{22}$, sendo determinada por interações intramoleculares quando o sistema está isolado, e por um efeito denominado cooperatividade ${ }^{23}$ quando o sistema está solvatado. Mesmo para um protótipo de ligação glicosídica para o qual foram calculados mapas conformacionais relaxados para o sistema isolado e em solução aquosa, as regiões de maior estabilidade encontradas foram exatamente as mesmas ${ }^{24}$ contrariando, portanto, a expectativa inicial de que a interação com o solvente pudesse originar estruturas que não fossem estáveis em fase gasosa.

Vale notar que a estrutura obtida se compara bastante bem com aquela recentemente relatada por Youngjin Choi et al. ${ }^{25}$, para a qual se verificou uma grande diferença de potencial eletrostático entre as partes superior e inferior dos anéis monossacarídicos.

Entretanto, existem outros graus de liberdade na molécula que ainda necessitam de investigação, para melhor identificar o número de estruturas estáveis na temperatura de 298,15 K. Dentre estes graus, em hierarquia na determinação das energias relativas, aquele que imediatamente se segue à orientação da ligação glicosídica, segundo nosso estudo, é a orientação dos grupos hidroximetilênicos.

Tabela 2. Confôrmeros com geometrias otimizadas em diferentes fases sem qualquer restrição. Os ângulos diedros estão em graus e as respectivas energias relativas eletrônicas e de Gibbs padrão em kcal/mol, $\mathrm{p}_{\mathrm{i}}$ (população relativa) está em \%

\begin{tabular}{|c|c|c|c|c|c|c|}
\hline \multicolumn{7}{|c|}{ Fase gasosa com geometria otimizada } \\
\hline Confôrmero & $\Phi$ & $\Psi$ & $\Delta \mathrm{U}_{\mathrm{T}=0 \mathrm{~K}}$ & $\Delta \mathrm{G}^{\mathrm{corr}}$ & $\Delta \mathrm{G}_{298 \mathrm{~K}}^{0}$ & $\mathrm{p}_{\mathrm{i}}^{\text {gas }}$ \\
\hline A & 81,6 & 78,1 & 0 & 220,7 & $0^{298 \mathrm{~K}}$ & 100 \\
\hline $\mathrm{C}$ & 179,2 & 179,3 & $+4,0$ & 222,0 & $+5,3$ & 0 \\
\hline \multicolumn{7}{|c|}{ Solução aquosa (modelo PCM) com geometria otimizada } \\
\hline Confôrmero & $\Phi$ & $\Psi$ & $\Delta \mathrm{G}_{\mathrm{T}=0 \mathrm{~K}}$ & $\Delta \mathrm{G}^{\mathrm{corr}}$ & $\Delta \mathrm{G}_{298 \mathrm{~K}}^{0}$ & $\mathrm{p}_{\mathrm{i}}^{\text {sol }}$ \\
\hline A & 75,2 & 75,6 & 0 & 217,6 & 0 & 100 \\
\hline $\mathrm{C}$ & 182,1 & 175,8 & $+6,8$ & 218,7 & $+7,9$ & 0 \\
\hline \multicolumn{7}{|c|}{ Cálculo "single point" em solução aquosa com geomeria da fase gasosa } \\
\hline Confôrmero & $\Phi$ & $\Psi$ & $\Delta \mathrm{G}_{\mathrm{T}-0 \mathrm{~K}}$ & $\Delta \mathrm{G}^{\mathrm{corr}}$ & $\Delta \mathrm{G}^{0}{ }_{298 \mathrm{~K}}$ & $\mathrm{p}_{\mathrm{i}}^{\text {sol }}$ \\
\hline A & 81,6 & 78,1 & $0^{1=0}$ & 227,5 & $0^{298 \mathrm{~K}}$ & 99,9 \\
\hline $\mathrm{C}$ & 179,2 & 179,3 & $+5,0$ & 227,0 & $+4,5$ & 0,1 \\
\hline
\end{tabular}

1) Em fase gasosa, para o confôrmero $A, U_{T=0 K}=-1290,715978$ u.a e $G_{298 K}^{0}=-1290,364328$ u.a., sendo $\Delta U_{T=0 K}=U_{T=0 K}(X)-U_{T=0 K}(A), \Delta G^{c o r r}=$ $\mathrm{G}^{0}{ }_{298 \mathrm{~K}}(\mathrm{X})-\mathrm{U}_{\mathrm{T}=0 \mathrm{~K}}(\mathrm{X})$ e $\Delta \mathrm{G}^{0}{ }_{298 \mathrm{~K}}=\mathrm{G}_{298 \mathrm{~K}}^{0}(\mathrm{X})-\mathrm{G}^{0}{ }_{298 \mathrm{~K}}(\mathrm{~A})$. 2) Em solução aquosa, para o confôrmero $\mathrm{A}, \mathrm{G}_{\mathrm{T}=0 \mathrm{~K}}=-1290,754701$ u.a. e $\mathrm{G}_{298 \mathrm{~K}}=-$ 1290,407998 u.a. sendo $\Delta \mathrm{G}_{\mathrm{T}=0 \mathrm{~K}}=\mathrm{G}_{\mathrm{T}=0 \mathrm{~K}}(\mathrm{X})-\mathrm{G}_{\mathrm{T}=0 \mathrm{~K}}(\mathrm{~A}), \Delta \mathrm{G}^{\text {corr }}=\mathrm{G}^{0}{ }_{298 \mathrm{~K}}(\mathrm{X})-\mathrm{G}_{\mathrm{T}=0 \mathrm{~K}}(\mathrm{X})$ e $\Delta \mathrm{G}^{0}{ }_{298 \mathrm{~K}}=\mathrm{G}_{298 \mathrm{~K}}^{0}(\mathrm{X})-\mathrm{G}_{298 \mathrm{~K}}^{0}(\mathrm{~A})$. 3) Cálculo "single point": para o confôrmero $A, G_{T=0 K}=-1290,743943$ u.a., sendo as correções térmicas e entrópicas as mesmas obtidas para a fase gasosa, somadas aos termos não-eletrostáticos. 
A investigação deste grau de liberdade é descrita a seguir.

Através da rotação dos grupamentos hidroximetilênicos em torno da ligação $\sim \mathrm{C}_{5}-\mathrm{C}_{6} \sim$ (Figura 1) para o confôrmero mais abundante obtido em solução aquosa (A), foram realizadas otimizações de geometria em fase gasosa para as nove possíveis orientações destes grupamentos: GGGG, GGGT, GGTG, GTGG, GTGT, GTTG, TGGG, TGGT e TGTG, cujos dados relevantes são mostrados na Tabela 3. Nesta nomenclatura, G e T significam gauche e trans, respectivamente; denotando $\mathrm{G}$ a orientação de $\mathrm{O}_{6}$ com respeito a $\mathrm{O}_{5}$, que vem definida pelo diedro $\omega\left(\mathrm{O}_{6}-\mathrm{C}_{6}-\mathrm{C}_{5}-\mathrm{O}_{5}\right)=60^{\circ}$ e $\mathrm{T}$ a orientação deste com respeito a $\mathrm{C}_{4}$ definida por $\omega\left(\mathrm{O}_{6}-\mathrm{C}_{6}-\mathrm{C}_{5}-\mathrm{C}_{4}\right)=180^{\circ 26}$.

Tabela 3. Confôrmeros com as geometrias otimizadas em fase gasosa para as nove possíveis orientações do grupamento hidroximetilênico. Os ângulos diedros estão em graus e as respectivas energias relativas eletrônica $\left(\mathrm{U}_{\mathrm{T}=0 \mathrm{~K}}\right)$ e de Gibbs padrão $\left(\mathrm{G}^{0}{ }_{298 \mathrm{~K}}\right)$ em kcal/mol, $\mathrm{p}_{\mathrm{i}}$ (população relativa) em \% e momento de dipolo $(\mu)$ em Debye

\begin{tabular}{lcccccc}
\hline Confôrmero & $\Phi$ & $\Psi$ & $\Delta \mathrm{U}_{\mathrm{T}=0 \mathrm{~K}}$ & $\Delta \mathrm{G}^{0}{ }_{298 \mathrm{~K}}$ & $\mathrm{p}_{\mathrm{i}}^{\text {gas }}$ & $\mu$ \\
\hline GGGG & 81,7 & 76,3 & $+2,8$ & $+2,2$ & 1,1 & 4,6770 \\
GGGT & 77,0 & 73,7 & $+0,8$ & $+0,5$ & 19,0 & 7,7032 \\
GGTG & 82,1 & 79,1 & $+4,1$ & $+3,8$ & 0,08 & 5,6047 \\
GTGG & 73,2 & 92,2 & $-0,5$ & $+0,5$ & 18,7 & 8,8347 \\
GTGT & 71,9 & 90,1 & $+3,6$ & $+3,9$ & 0,07 & 5,9472 \\
GTTG & 73,3 & 93,0 & $+3,9$ & $+4,6$ & 0,02 & 6,7298 \\
TGGG & 81,3 & 75,4 & 0 & 0 & 44,6 & 4,8147 \\
TGGT & 75,6 & 66,7 & $+0,8$ & $+0,7$ & 13,8 & 5,0228 \\
TGTG (A) & 81,6 & 78,1 & $+1,4$ & $+1,6$ & 2,8 & 6,4245 \\
\hline
\end{tabular}

Para o confôrmero TGGG, $\mathrm{U}_{\mathrm{T}=0 \mathrm{~K}}=-1290,718275$ u.a e $\mathrm{G}^{0}{ }_{298 \mathrm{~K}}=$ $-1290,366938$ u.a., sendo $\Delta \mathrm{U}_{\mathrm{T}=0 \mathrm{~K}}=\mathrm{U}_{\mathrm{T}=0 \mathrm{~K}}(\mathrm{X})-\mathrm{U}_{\mathrm{T}=0 \mathrm{~K}}(\mathrm{TGGG}) \mathrm{e}$ $\Delta \mathrm{G}_{298 \mathrm{~K}}^{0}=\mathrm{G}_{298 \mathrm{~K}}^{0}(\mathrm{X})-\mathrm{G}_{298 \mathrm{~K}}^{0}(\mathrm{TGGG})$.

O cálculo de população de Boltzmann $\left(\mathrm{p}_{\mathrm{i}}\right)$ para estes nove confôrmeros da trealose em fase gasosa também é apresentado na Tabela 3.

Considerando as possibilidades de orientação dos grupos hidroximetilênicos, observamos que, na realidade, seis estruturas (GGGG, GGGT, GTGG, TGGG, TGGT e TGTG) têm população maior que $1 \%$, para a molécula isolada em $298,15 \mathrm{~K}$, sendo TGGG a mais abundante.

Para aqueles confôrmeros com valor de $\Psi$ em torno de $90^{\circ}$ observou-se a formação de uma ligação hidrogênio intramolecular entre os átomos de $\mathrm{O} 6$ (hidroximetilênico) e H2' (ligado ao átomo C2' do anel), possível para todas as orientações GTGG, GTGT e GTTG. É importante salientar que nenhuma destas estruturas é aquela mais estável mostrando, portanto, que a justificativa de estabilidade relativa entre confôrmeros de dissacarídeos por modelos qualitativos pode ser extremamente falha.

Através da solvatação (com o modelo PCM) dos nove confôrmeros otimizados em fase gasosa, foram obtidas as geometrias e dados de energia que estão na Tabela 4.

A partir dos dados da Tabela 4, observamos que a interação com um solvente como a água, de alta constante dielétrica $(\varepsilon=78,39)$, parece favorecer basicamente a existência de somente duas estruturas, que são exatamente aquelas com maior momento de dipolo, embora aquela bem mais abundante não seja a mais polar. Novamente as estruturas com valor de $\Psi$ em torno de $90^{\circ}$ estabelecem ligação hidrogênio intramolecular entre os átomos de O6 (hidroximetilênico) e H2' (ligado ao átomo C2' do anel). Estas estruturas são as mesmas presentes em fase gasosa: GTGG,GTGT e GTTG, além da estrutura TGGT, sendo a primeira mostrada na Figura 4, por ser a única deste conjunto representativa (pouco) em solução.
Tabela 4. Confôrmeros reotimizados para a trealose em solução aquosa, considerando a orientação dos grupamentos hidroximetilênicos. Os ângulos diedros estão em graus e as respectivas energias relativas livre em solução considerando e não considerando os fatores térmicos e entrópicos $\left(\Delta \mathrm{G}_{\mathrm{T}=0 \mathrm{~K}}\right.$ e $\Delta \mathrm{G}_{298 \mathrm{~K}}$, respectivamente) em $\mathrm{kcal} / \mathrm{mol}, \mathrm{p}_{\mathrm{i}}$ (população relativa) em $\%$ e $\mu$ em Debye

\begin{tabular}{lcccccc}
\hline Confôrmero & $\Phi$ & $\Psi$ & $\Delta \mathrm{G}_{\mathrm{T}=0 \mathrm{~K}}$ & $\Delta \mathrm{G}^{0}{ }_{298 \mathrm{~K}}$ & $\mathrm{p}_{\mathrm{i}}^{\text {sol }}$ & $\mu$ \\
\hline GGGG & 75,9 & 77,6 & $+9,5$ & $+10,0$ & $4,3 \times 10^{-6}$ & 6,1400 \\
GGGT & 72,7 & 77,8 & 0 & 0 & 99,5 & 9,8344 \\
GGTG & 74,5 & 78,9 & $+8,4$ & $+8,9$ & $3,0 \times 10^{-5}$ & 8,3248 \\
GTGG & 73,6 & 94,4 & $+4,3$ & $+3,2$ & 0,5 & 10,8867 \\
GTGT & 71,4 & 93,7 & $+9,1$ & $+7,4$ & $4,0 \times 10^{-4}$ & 7,3863 \\
GTTG & 72,7 & 96,8 & $+6,5$ & $+8,2$ & $9,3 \times 10^{-5}$ & 9,3922 \\
TGGG & 76,9 & 78,3 & $+6,4$ & $+7,5$ & $3,0 \times 10^{-4}$ & 6,3177 \\
TGGT & 69,3 & 93,3 & $+8,5$ & $+8,6$ & $5,1 \times 10^{-5}$ & 7,4342 \\
TGTG & 79,3 & 77,9 & $+4,2$ & $+5,3$ & 0,01 & 9,7233 \\
\hline
\end{tabular}

Para o confôrmero GGGT, $\mathrm{G}_{\mathrm{T}=0 \mathrm{~K}}=-1290,762474$ u.a e $\mathrm{G}_{298 \mathrm{~K}}^{0}=$ $-1290,421040$ u.a., sendo $\Delta \mathrm{G}_{\mathrm{T}=0 \mathrm{~K}}=\mathrm{G}_{\mathrm{T}=0 \mathrm{~K}}(\mathrm{X})-\mathrm{G}_{\mathrm{T}=0 \mathrm{~K}}(\mathrm{GGGT})$ e $\Delta \mathrm{G}^{0}{ }_{298 \mathrm{~K}}=\mathrm{G}^{0}{ }_{298 \mathrm{~K}}(\mathrm{X})-\mathrm{G}^{0}{ }_{298 \mathrm{~K}}(\mathrm{GGGT})$.

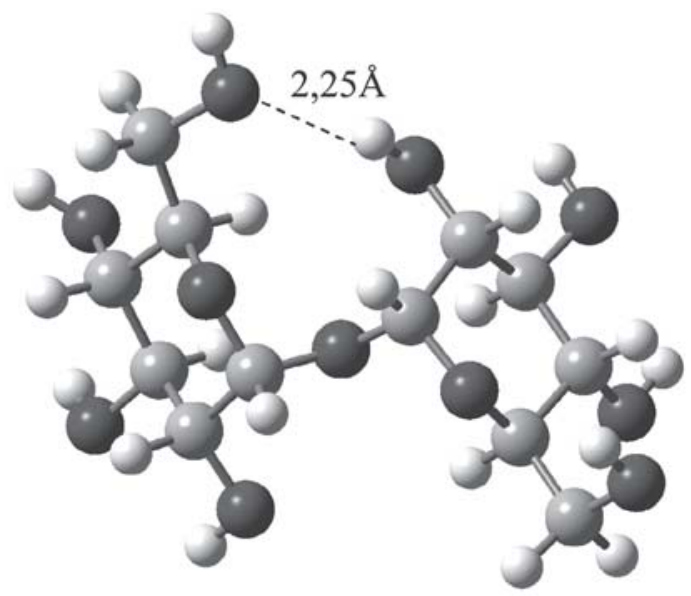

Figura 4. Trealose na conformação GTGG obtida em solução aquosa, onde há uma ligação hidrogênio intramolecular entre os átomos de O6 e H2'

A partir dos dados das Tabelas 3 e 4, observa-se que o conjunto formado por seis estruturas representativas para a trealose em fase gasosa (GGGG, GGGT, GTGG, TGGG, TGGT e TGTG) se reduz para uma estrutura (GGGT), praticamente, abundante em solução. Sendo que a estrutura mais abundante em fase gasosa (TGGG) não é aquela que se mantém em solução. Este resultado, juntamente com aqueles provenientes de estudos dos efeitos de solvatação sobre a estrutura e a energia separadamente (Tabela 2), nos permite concluir que os efeitos intramoleculares são determinantes na conformação da trealose, enquanto que os efeitos intermoleculares na determinação das energias relativas de seus confôrmeros.

Com base na Equação de Karplus, os valores das constantes de acoplamento de spin heteronuclear $\left({ }^{3} \mathrm{~J}_{H, C}\right)$ para cada um dos dois confôrmeros significativamente abundantes em solução aquosa foram calculados, e são apresentados na Tabela 5. Esta constante diz respeito ao acoplamento entre os átomos $\mathrm{H}$ e $\mathrm{C}$ da ligação glicosídica, como ilustrado na Figura 1 , onde ${ }^{3} \mathrm{~J}_{H, C}\left(\Phi_{\mathrm{H} 1}\right)$ é obtido para a sequiência $\Phi_{\mathrm{H} 1}=\mathrm{H} 1-\mathrm{C} 1-\mathrm{O}-\mathrm{C} 1{ }^{\prime} \mathrm{e}^{3} \mathrm{~J}_{H, C}\left(\Phi_{\mathrm{H} 1}\right)$ obtido para a seqüência $\Phi_{\mathrm{H} 1}=\mathrm{H} 1$ '-C1'-O-C1.

Para se estabelecer uma comparação com os valores experimentais de ${ }^{3} \mathrm{~J}_{H, C}$ encontrados na literatura, foi realizada uma mé- 
Tabela 5. Valores para os ângulos diedros (em graus) dos segmentos H-C-O-C da ligação glicosídica dos confôrmeros de trealose mais abundantes em solução aquosa e seus respectivos valores de ${ }^{3} \mathbf{J}_{H, C}(\mathrm{~Hz})$ individuais e médio

\begin{tabular}{lcc}
\hline Confôrmero & GGGT & GTGG \\
\hline$\Phi_{\mathrm{H} 1}$ & $-45,3$ & $-43,7$ \\
$\Phi_{\mathrm{H} 1^{\prime}}\left(\Phi_{\mathrm{H} 1}\right)$ & $-40,0$ & $-22,7$ \\
${ }^{3} \mathrm{~J}_{H, C}\left(\Phi^{\prime}\right)$ & 2,9 & 3,0 \\
${ }^{3} \mathrm{~J}_{H, C}\left(\Phi_{\mathrm{H} 1}\right)$ & 3,4 & 4,8 \\
\hline${ }^{3} \mathrm{~J}_{H, C}\left(\Phi_{\mathrm{H} 1}\right)$ médio & 2,9 \\
${ }^{3} \mathrm{~J}_{H, C}\left(\Phi_{\mathrm{H} 1}\right)$ médio $^{27}$ & 3,4 \\
Outro trabalho & 2,4 \\
Experimental & $3,3^{28}-3,19(0,05)^{29}$ \\
\hline
\end{tabular}

dia, ponderada pela população de Boltzmann, destes valores obtidos para cada confôrmero em solução aquosa. Os resultados são também mostrados na Tabela 5, juntamente com os valores de, ${ }^{3} \mathrm{~J}_{H, C}, \Phi_{\mathrm{H} 1}$ e $\Phi_{\mathrm{H} 1}$, encontrados na literatura para estruturas obtidas por diferentes metodologias. Os resultados sugerem que a estrutura obtida em solução apresenta um valor de ${ }^{3} \mathrm{~J}_{H, C}$ que se compara bastante satisfatoriamente com aquele experimental podendo, portanto, responder pela conformação da trealose em solução aquosa, para o sistema a $298,15 \mathrm{~K}$.

\section{CONCLUSÃO}

A fim de determinar os confôrmeros mais abundantes da trealose em solução aquosa na temperatura de $298,15 \mathrm{~K}$, cálculos de otimização de geometria foram realizados em nível HF/6-31G(d,p) para os cinco confôrmeros da trealose identificados como mínimos de energia num mapa conformacional construído previamente para a molécula isolada.

Estas cinco estruturas, quando seus parâmetros geométricos foram completamente otimizados, resultaram na realidade em somente duas estruturas estáveis tanto no vácuo como em solução aquosa, que mantêm seus ângulos glicosídicos independentemente da natureza da fase em que se encontram, revelando que são os efeitos intramoleculares, e não aqueles intermoleculares (interação com o solvente), que determinam suas conformações.

A partir do confôrmero mais abundante em solução aquosa as orientações dos grupamentos hidroximetilênicos foram investigadas, ou seja, um segundo parâmetro geométrico foi considerado a fim de se observar sua influência na energia de uma dada conformação.

Nesta investigação, seis estruturas, dentre as nove possíveis, foram obtidas com população maior que $1 \%$ para a molécula isolada, enquanto uma somente foi obtida em solução aquosa.

Este resultado, juntamente com outros anteriormente discutidos, nos permite concluir que os efeitos intramoleculares são determinantes na conformação da trealose, enquanto que os efeitos intermoleculares na determinação da abundância relativa de seus confôrmeros.

Para esta estrutura em solução, foi calculado o valor de ${ }^{3} \mathbf{J}_{H, C}$, que muito bem se compara com aquele experimental, validando, portanto, tal geometria.

Estudos da interação da trealose com um protótipo de membrana fosfolipídica estão em progresso.

\section{AGRADECIMENTOS}

Às agências CNPq e FAPERJ pelo apoio financeiro concedido a este trabalho.

\section{REFERÊNCIAS}

1. Berg, J. M.; Tymoczko, J. L.; Stryer, L.; Bioquímica, 5a ed., Guanabara Koogan: Rio de Janeiro, 2004.

2. Elbein, A. D.; Adv. Carbohyd. Chem. Biochem. 1974, 30, 227.

3. Argüelles, J. C.; Arch. Microbiol. 2000, 174, 217.

4. Herdeiro, R. S.; Pereira, M. D.; Panek, A. D.; Eleutherio, E. C. A.; Biochem. Biophys. Acta, Gen. Subj. 2006,1760, 340.

5. Cerrutti, P.; de Huergo, M. S.; Galvagno, M.; Schebor, C.; Buera M. D.; Appl. Microbiol. Biotechnol. 2000, 54, 575.

6. Pereira, C. S.; Lins, R. D.; Chandrasekhar, I.; Freitas, L. C. G.; Hünenberger, P. H.; Biophys. J. 2004, 86, 2273.

7. Sum, A. K.; Faller, R.; de Pablo, J. J.; Biophys. J. 2003, 85, 2830.

8. Doxastakis, M.; Sum, A. K.; de Pablo, J. J.; J. Phys. Chem. B 2005,109, 24173.

9. Sum, A. K.; Chem. Biodiversity 2005, 2, 1505.

10. Skibinsky, A.; Venable, R. M.; Pastor, R. W.; Biophys. J. 2005, 89, 4111.

11. Csonka, G.; J. Mol. Struct. (Theochem) 2002, 584, 1.

12. Silva, C. O.; Theor. Chem. Acc. 2006, 116, 137.

13. Silva, C. O.; Nascimento, M. A. C.; Carbohydr. Res. 2004, 339, 113.

14. Hemmingsen, L.; Madsen, D. E.; Esbensen, A. L.; Olsen, L.; Elgensen, S. B.; Carbohydr. Res. 2004, 339, 937.

15. Pérez, S.; Imberty, A.; Elgensen, S. B.; Gruza, J.; Mazeau, K.; JiménezBarbero, J.; Poveda, A.; Espinosa, J-F.; van Eyck, B. P.; Johnson, G.; French, A. D.; Kouwijzer, M. L. C. E.; Grootenius, P. D. J.; Bernardi, A.; Raimondi, L.; Senderowitz, H.; Durier, V.; Vergoten, G.; Rasmussen, K.; Carbohydr. Res. 1998, 314, 141.

16. Polygraf v3.2.1, Molecular Simulations Inc., 1992.

17. Jaguar 6.0, Schrödinger Inc., Porland, OR, USA.

18. Gaussian 03, Revision B.04; Frisch, M. J.; Trucks, G. W.; Schlegel, H. B.; Scuseria, G. E.; Robb, M. A.; Cheeseman, J. R.; Montgomery Jr.; J. A., Vreven, T.; Kudin, K. N.; Burant, J. C.; Millam, J. M.; Iyengar, S. S.; Tomasi, J.; Barone, V.; Mennucci, B.; Cossi, M.; Scalmani, G.; Rega, N.; Petersson, G. A.; Nakatsuji, H.; Hada, M.; Ehara, M.; Toyota, K.; Fukuda, R.; Hasegawa, J.; Ishida, M.; Nakajima, T.; Honda, Y.; Kitao, O.; Nakai, H.; Klene, M.; Li, X.; Knox, J. E.; Hratchian, H. P.; Cross, J. B.; Adamo, C.; Jaramillo, J.; Gomperts, R.; Stratmann, R. E.; Yazyev, O.; Austin, A. J.; Cammi, R.; Pomelli, C.; Ochterski, J. W.; Ayala, P. Y.; Morokuma, K.; Voth, G. A.; Salvador, P.; Dannenberg, J. J.; Zakrzewski, V. G.; Dapprich, S.; Daniels, A. D.; Strain, M. C.; Farkas, O.; Malick, D. K.; Rabuck, A. D.; Raghavachari, K.; Foresman, J. B.; Ortiz, J. V.; Cui, Q.; Baboul, A. G.; Clifford, S.; Cioslowski, J.; Stefanov, B. B.; Liu, G.; Liashenko, A.; Piskorz, P.; Komaromi, I.; Martin, R. L.; Fox, D. J.; Keith, T.; Al-Laham, M. A.; Peng, C. Y.; Nanayakkara, A.; Challacombe, M.; Gill, P. M. W.; Johnson, B.; Chen, W.; Wong, M. W.; Gonzalez, C.; Pople, J. A.; Gaussian, Inc., Pittsburgh PA, 2003.

19. Cammi, R.; Tomasi, J.; J. Comput. Chem. 1995, 16, 1449; Mennucci, B.; Cancès, E.; Tomasi, J.; J. Chem. Phys. 1997, 107, 3032.

20. Tomasi, J.; Mennucci, B. ; Cammi, R.; Chem. Rev. 2005, 105, 2999.

21. Tvaroška, I.; Hricovini, M.; Petracova, E.; Carbohydr. Res. 1989, 189, 359.

22. Silva, C. O.; Vreven, T.; Mennucci, B.; J. Org. Chem. 2004, 69, 8161.

23. Klein, R. A.; J. Am. Chem. Soc. 2002, 124, 13931.

24. Silva, C. O.; Nascimento, M. A. C.; Theor. Chem. Acc. 2004, 112, 342.

25. Choi, Y.; Cho, K. W.; Jeongc, K.; Jungd, S.; Carbohydr. Res. 2006, 341, 1020 .

26. Bitzer, R. S.; Barbosa, A. G. H.; Silva, C. O.; Nascimento, M. A. C.; Carbohydr. Res. 2005, 340, 2171.

27. Poveda, A.; Vicent, C.; Penades, S.; Jimenez-Barbero, J.; Carbohydr. Res. 1997, 301, 5.

28. Batta, G.; Kover, K. E.; Gervay, J.; Hornyak, M.; Roberts, G. M.; J. Am. Chem. Soc. 1997,119, 1336.

29. Norman, W. H. C.; Paramita, D.; Graham, E. B.; Carbohydr. Res. 2003, 338,955 . 